\title{
Design and Development of Aircraft to Aircraft Communication by Ad-Hoc Networks
}

\author{
Vengadesh.A ${ }^{1}$, Gunasekaran.P2 \\ 1\&2PSN College of Engineering and Technology \\ 1iaivenky1991@gmail.com, 2mailtogunasekar@gmail.com
}

\section{Abstract-}

This paper is designed to develop an air to air navigation like aircraft to aircraft communication (A2AComm) based on Ad-Hoc networks as a communication technology. It is a new communication framework of Ad-hoc network for safe air navigation. The main principle Ad-hoc network is that, it will track the position of nearby aircrafts with motion, direction and it will establish communication between two or more adjacent flying aircrafts. The design of A2AComm is mainly to avoid air traffic congestion, pointing emergency location, reducing the Air Traffic Control (ATC) workloads and preventing aircraft diversion and air accidents. Also its an easy mode of communication between crews from one aircraft to another aircraft using audible and visual alerts. Development of this design is not based on Global Positioning System (GPS) but fully based on WiMAX and Ad-hoc network principle. Ad-Hoc network communication covers a maximum distance of 1000 meters $(1 \mathrm{Km})$ and has a high bandwidth and high rate of data transmission. The systems comprise computers, communications, sensors, control technologies and management strategies. These function in an integrated manner to improve the functioning of the air transportation systems. It also provides real-time information to the aircrew to improve the safety and efficiency of air to air communication.

Keywords-A2A Comm, Ad-Hoc Networks, WiMax, Cockpit Display, Traffic Control.

\section{INTRODUCTION}

An air-miss is defined as an incident associated with an operation of an aircraft in which a possibility of collision occurs as a result of close proximity of less than 500 feet to another aircraft or a report is received from a pilot or a flight crew member stating a collision hazard existed between two or more aircraft.

Air-miss leads to mid air collision. Owing to the relatively high rate velocities involved and a subsequent impact on the ground or sea level, very severe damage or total destruction of at least one of the aircraft involved usually results. The reason for a mid-air collision is increased by miscommunication, mistrust, error in navigation, and deviations from approved flight path. Although a rare occurrence due to the very large air space available, collisions often happen in the vicinity or at airports locality, due to the large volume of flights operating and limited air space when compared to general flight.[1]

The aviation industry is developing rapidly to occupy the increasing demand for faster, comfortable and safe transportation. Air traffic level is a critical phase and high accuracy is required especially when flying under constraints of miscommunication, navigation error and flight plan deviation conditions. The zero accident policy announced by Federal Aviation Authority (FAA) requires airliners to have essentially perfect navigation from takeoff to landing.

It requires safe transportation in conditions of no visibility or altitude visual range. Currently, the limits of real-time communication and standards of data transmission equipments have not been able to match ICAO specifications, standards and recommended practices.

Despite, these are still in use due to the lack of better alternatives. The main current equipment limitations are: inaccuracy, mistrust, communication error, obstruction in signal broadcasting, delay of ATC commands, lack of integrity and high cost. This paper presents a new approach to the communication system that meets ICAO standards (i.e.) improve the craft to craft real-time communication, high rate data transmission, avoid mid-air collision thus leading to enhanced flight safety. 


\section{SYSTEMS OVERVIEW}

Especially for aircraft to aircraft inter-link communication related and present systems are RADAR tracking, ATC to Aircraft Communication, Automatic dependent surveillance-broadcast (ADS-B), Portable Collision Avoidance System (PCAS), Traffic Collision Avoidance System (TCAS), High Frequency (HF), Very High Frequency (VHF), Global Positioning System (GPS), and Differential Global Positioning System (DGPS). This system information are not fully able to match the ICAO standards. Currently, GPS is the main navigation system used all over the world for aircraft navigation, approach and communication.

However, in aircraft approach and communication, the accuracy of GPS is not sufficient to perform a perfect pointing and positioning due to the possibility of aircraft to be drifted out of [2]. Also these systems are difficult to maintenance and high cost.

The other GPS limitations are: Satellite unavailability, Satellite Geometry, Low vertical accuracy, Satellite signal broadcasting travel time is longer than signals transmitted from ground surface, GPS receiver update rate is low, Signal weakening and degradation, lonosphere effects, GPS lack of high accuracy [3].

In order to overcome GPS limitations, even though many systems have been designed to augment GPS and improve the accuracy, no system can be relied on to achieve a high accuracy in a range of less than $1 \mathrm{~m}$ for high speed applications with high integrity and reliability [4].

A2A Comm by Ad-Hoc network based display system proposes an animation and/or automatic information about airplane position, altitude and its displays all information, related aircraft flying in any weather conditions. This system is not based on GPS; it depend only WiMax and Ad-Hoc network principle and displays the parameters as per gathering information and transfer data via WiMax. This proposed system designed for increase the accuracy, real-time communication between the aircrafts; reduce the ATC and pilots work load and safety transportation. The design of A2A Comm by Ad-Hoc networks based system shown in Figure 1 . This A2A Comm by Ad-Hoc network based system concept is based on WiMax range coverage and Ad-Hoc principle information's as per airplane models/types.

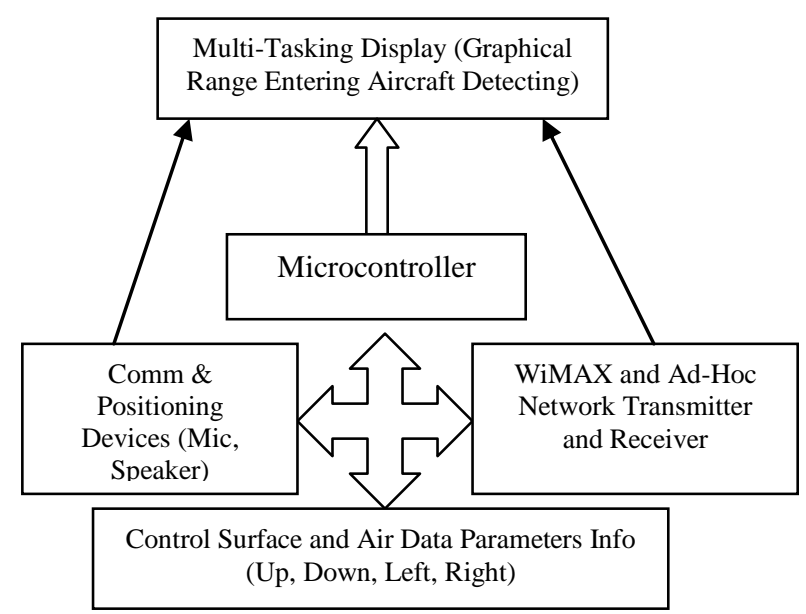

Fig.1: Functional block diagram of A2A Comm based Cockpit Display

\section{SYSTEM OPERATIONS}

\section{A. POSITIONING AND COMMUNICATION OPERATION}

This A2A Comm by Ad-Hoc network based system is proposed to be used for aircraft to aircraft real-time communication, data transmission and exchange diversion commands where the area is considered two and more airplanes comes in a same flight path or near flying air surface. So a WiMax technology which is advance of wireless technology could be used to cover the $1000 \mathrm{~m}$ of aircraft around area for airplanes pointing and position. The chosen technology should be in a high range and band width where the signal propagation is not influenced by weather phenomena like, rain, snow or clouds. The characteristics of WiMax propagation are ideal for long range data transmission. It does not interfere with transmissions thousands of kilometres away. Therefore, it is less affected by atmospheric noise and interference from electrical equipment than lower frequencies [5]. WiMAX spectrum ranges from 10 to 66 $\mathrm{GHz}$ are used for A2A Communication and Data transmission.

The selected Ad-Hoc multi-hop network via WiMAX pre-set coverage range is important consideration for to cover the $1000 \mathrm{~m}$ around the selected aircraft. In this system, the data's are broadcasted to receiver via any 
range from Ad-Hoc Transmitter (Tx) node. Ad-Hoc Receiver ( $R x)$ node on the airplanes receives the transmitted data's or information from range entering aircraft or same flight path aircraft and measures the exact aircraft position, direction and altitude with the help of pilot to pilot WiMAX communication through this system [6]. The location of the nearby aircraft is pointed before the 1000meters in end of the WiMAX coverage. In this cockpit display, emergency point is marked as circular line and it indicate the distances in meter. Here circular shapes are mentioned in red colour line and it indicates that total area of circle is 1 kilo meters (1000 meters). Based on aircraft movement distance red lines will vary. The WiMAX signal coverage area is marked as red colour and it shows the signal coverage area and it depends on band width range. The received signal range can be obtained by Equation (1).

$$
\text { Range }=(\text { speed }) \times(\text { Time })
$$

Where the speed of the signal is the speed of light and time is the time that the signal takes between transmitter and receiver [7]. Then receiver can calculate the coordinates (longitude and altitude) of the airplanes, depending on the received signal strength ranges.

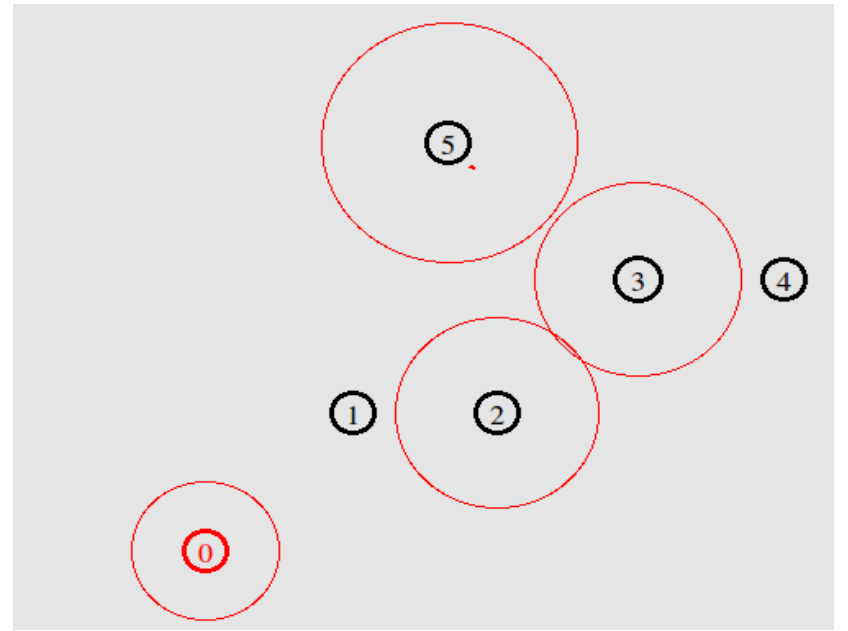

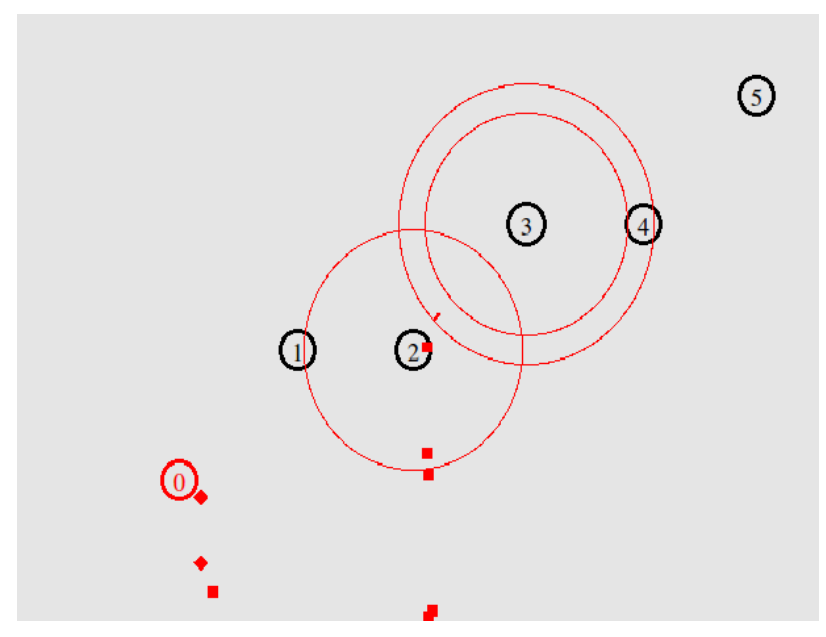

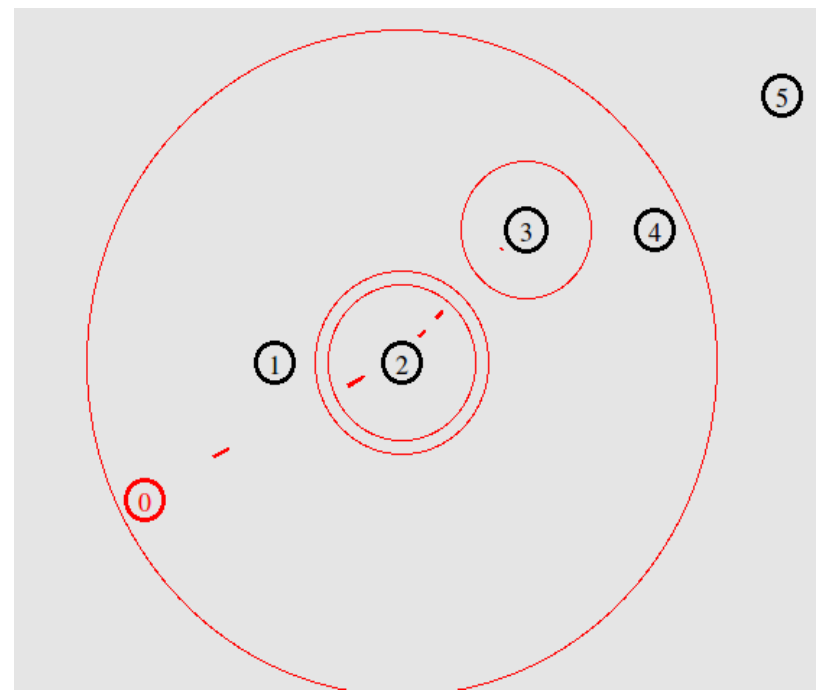

Figure 2: Sample A2A NS-2 Output

\section{B. COCKPIT DISPLAY}

Cockpit display is based on LCD screen, because it will consume less power consumption and better brightness. In this A2A based cockpit display has installed Network Simulator-2(ns-2) programmed device with coded operational process. This display divided into four parts and displays different parameters like WiMAX range and power plant, Aircraft positioning related warning information's, Control surfaces parameters and live animation about airplanes movements according to real observing information's from sources. Airplanes movements and positioning information's like flying distances (Preset WiMAX total distance, line cross point location and remaining distance), Altitude, Direction, Airplanes symbol movement and Commands are displayed in centre part of the A2A Comm based Cockpit 
display. These sets of cockpit display shown in below Figure 3.

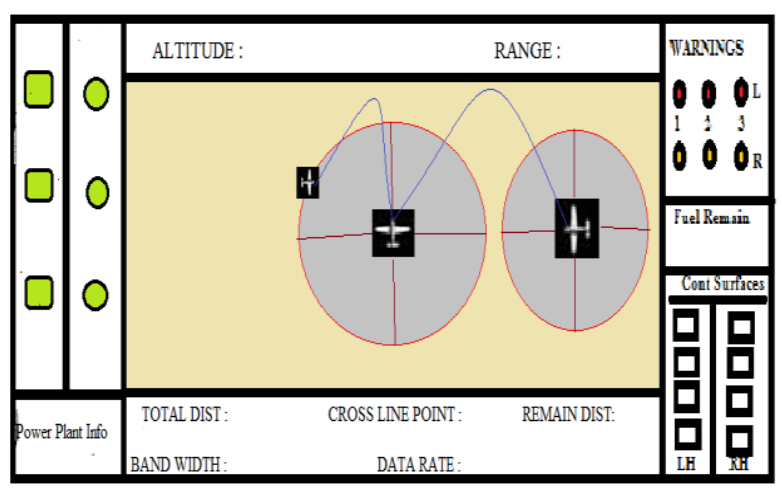

Figure 3: A2A Comm based Cockpit Display

In Power plant parameters like Engine speed, Fuel flow Rate, Inter Turbine Temperature (ITT), Oil Temperature, Fuel and Oil flow Pressures of the both sides ( $\mathrm{LH}$ and $\mathrm{RH}$ ) of engines are shown in Left side of cockpit display. This all information is mentioned total six shapes in display shown in above the figure. Right side of the cockpit display divide into three sectional parts and are displays only waring information's. Warning information's is flying related important parameters in Power plant, Fuel quantity and Control surfaces. All warning indications are like LED glowing and it will vary different colours. Mostly three colours are used to intimate the warning information.

If any of the parameter is in critical condition means it display will shows RED colour and normal condition means GREEN colour. Before the critical condition means display will shows YELLOW or AMBER colour. This all operations are controlled by Microcontroller kit and display operations and designs are based on Visual Basic and National Instruments. Fuel quantity also mentioned and it will shows in digital format. All these waring parameters are arranged in as per primary and secondary controller parameters.

\section{SYSTEM CONTROL INFORMATION}

\section{a) Safety Application}

WiMAX based Ad-Hoc network can significantly reduces the lag in shows emergency warning [8]. This system installed aircrafts can exchange data's and messages to each other about directional operations and difficulties in flying. An aircraft can able to gather a dangerous situation and is suddenly intimate or alert the range entering or line cross aircrafts. This system allows a faster reaction to the emergency situation. The warning message information's are comes from the ad-hoc nodes via WiMAX. The air traffic information can also be sent.

\section{b) Privacy in WiMAX based Ad-Hoc Network}

According to privacy, which is allows for a aircraft to communicate with range entering other aircraft do not disclosing it's actual identity [9][10]. This security achieved by aircraft masking for retain the permanent identity, so that it won't known the other private aircraft.

\section{c) WiMAX Range Propagation}

The A2A Comm by Ad-Hoc network system has used WiMAX IEEE 802.16 standards for communication and data transmission. This standard was developed to standardize the 'last mile' wireless distribution technology. This technology will provide fixed, nomadic, portable and mobile wireless broadband connectivity without the need for a direct line-of-sight.

For A2A Comm system has used licensed signal technology. WiMAx operates same as WiFi principles and it transfer data's from one node to another node via radio signals. Here cockpit display equipped with WiMAX would receive data from the range entering aircraft WiMAX transmitting station, probably using encrypted data keys to prevent unauthorized users from stealing access. This system can transmits the data range of 30 mile $(50 \mathrm{~km})$ radius from the base station, but we preset only 1000 meters $(1 \mathrm{~km})$ for comfort transmission and data transfer speed is up to 70 megabits per second. WiMAX frequency bands of 2 to $11 \mathrm{GHz}$ and 10 to $66 \mathrm{GHz}$ (licensed and unlicensed band). Here we used only licensed band for security reasons.[11]

\section{d) Microcontroller}

PIC based microcontrollers are provide best performance for control system operations. In A2A Comm based cockpit display, microcontroller is major part of the instrument section. It will control the display the information's based on gathering data or information's from source [12]. Mostly it will used to control the warning information's and control surface parameters. Also it will 
control the command words as per user conditions (i.e. if any one of the parameter is not in condition means, commands will displays). Commands are used for to understand the status about aircraft flying conditions and operation level. Commands in displays would be helpful in reducing flight deck workload and ensuring safety especially by reducing the scan time for searching all parameters.

Based on the collected information's form source, the commands display will brings challenges such as potential increase in error rate, loss of sensory feedback, and difficult operation during confusion. In order to evaluate the effectiveness of interfaces depends on ICAO standards and designer of the airplanes. Microcontroller will observe the data's from source and it performs as per user codes [13]. All codes are based on C, C++ and OTcl are in ns-2 simulation Technologies.

\section{A2A Comm AdvantAges}

This system is proposed to overcome the majority of aircraft real-time communication and high rate data transmission limitations. It has many advantages over current systems. The main purpose of this study is to design a system to reduce some positioning errors and eliminate others. This study aims to meet ICAO standard for the ability to real-time communication, data transmission and track the same flight path travelling aircraft in bad weather and low visibility conditions. In addition, this system gives the real and accurate altitude and position of the range entering aircraft since it refers to the radar, ATC command and GPS system. Moreover, the signal broadcasted from satellites takes about 0.07 second to reach to earth whereas it takes about 0.000004 second if the signal is transmitted from earth surface. Besides that, signal transmitted from Ground-based system will be weaker.

Additionally, satellites unavailability due to satellites distribution or construction blockage is a serious problem in critical stages of same flight path aircraft positioning or tracking, this system is designed to be available continuously within the communication and flying area. Also, due to the distribution of satellites the vertical accuracy is always less than horizontal accuracy. More than that it causes an accuracy reduction when the visible satellites are all clustered together in a single quadrant, this narrows satellites visibility angle and consequently reduce the positioning accuracy.

\section{CONCLUSIONS}

Aircraft to aircraft real-time communication and high rate data transmission is a very difficult and it risk for airplanes to be diverted out of the desired flight path. So a superior accuracy and real-time communication is required to guide the aircrafts from take-off to landing. In high rate air traffic conditions, when inter-linked craft communication range does not exist, aircraft will need GPS, RADAR or ATC commands systematically. Currently, these system are not that much able to provide this reliability all over the world. In this system has been proposed to reduce and eliminate the mid-air collision and air traffic consequences. This System characteristic is to enhance the craft to craft communication and data transmission in all weathers safely with high accuracy guidance.

Overall, this system is completely autonomous. It does not depend on GPS satellites such as DGPS. This system provides an accurate airplane diversion from recommended route points and enhancing the airplanes to communicate in all level flying conditions. Finally, the system results show that integrity, visibility, availability, real-time communication and accuracy have been considerably increased.

\section{ACKNOWLEDGMENT}

I would like to express my gratitude to Department of Aeronautical Engineering Staff members in PSN College of Engineering and Technology (PSNCET) for this research oriented theoretical framework is made possible.

\section{REFERENCES}

[1]. http://en.wikipedia.org/wiki/Mid-air_collision?oldid=627452116.

[2]. http://en.wikipedia.org/wiki/Differential_GPS.

[3]. Honeywell Corporation, Combined vision system and GPS landing system for low visibility operation, China - US Aviation New Technology Workshop, Beijing, June 6 -7, 2012.

[4]. Hamed Sheikhpour, Gholamreza Shirazian and Ebrahim Safa, An Approach on Take-off and Landing Related Aircraft Accidents Involving New Considerations, IPCSIT vol. 26 , International Conference on Traffic and Transportation Engineering ,Singapore,2012.

[5]. Josiane Nzouonta, Neeraj Rajgure, Guiling (Grace) Wang, Member, IEEE, and Cristian Borcea, Member, IEEE, VANET 
Routing on City Roads Using Real-Time Vehicular Traffic Information, IEEE TRANSACTIONS ON VEHICULAR TECHNOLOGY, VOL. 58, NO. 7, SEPTEMBER 2009.

[6]. David Tse and Pramod Viswanath. Fundamentals of wireless communication. Cambridge University Press, New York, NY, USA, 2005.

[7]. Jerzy Mikulski, Advances in Transport Systems Telematics, Monograph. Publisher Chair of Automatic Control in Transport, Faculty of Transport, Silesian University of Technology. Katowice 2007.

[8]. Balmahoon R, Peplow R (Prof);, Computer Engineering, University of KwaZulu Natal, King George V Avenue, Glenwood, Durban, 404,21-'Vehicular Ad-Hoc Networks: An Introduction to Privacy- 2010".

[9]. Brijesh Kumar Chaurasia, Shekhar Verma, G. S. Tomar, and S.M. Bhaskar, "Pseudonym Based Mechanism for Sustaining
Privacy in VANETs," in First International Conference on Computational Intelligence, Communication Systems and Networks, 2009, pp. 420 - 425.

[10]. Matthias Gerlach and F Guttler, "Privacy in VANETs using Changing Pseudonyms - Ideal and Real," in IEEE 65th Vehicular Technology Conference, 2007, pp. 2521 - 2525.

[11]. ITU-T Rec. H.323, "Packet based Multimedia Communications Systems", v. 2, 1998.

[12]. Sridher Kaminani, Touch Screen Technology in Flight Deck, How Far Is It Helpful, 31st Digital Avionics Systems Conference, October 14-18, 2012.

[13]. Lynda J. Kramer, Steven P. Williams, Trey Arthur III, Sherri Rehfeld, and Stephanie J. Harrison ,Motion-Base Simulator Evaluation of an Aircraft Using an External Vision System, U.S. Government work not protected by U.S. copyright. 